\title{
Determinism, Physical Possibility, and Laws of Nature
}

\author{
Balázs Gyenis ${ }^{1,2}$ (1)
}

Received: 15 November 2019 / Accepted: 6 January 2020 / Published online: 21 January 2020

(c) The Author(s) 2020

\begin{abstract}
We call attention to different formulations of how physical laws relate to what is physically possible in the philosophical literature, and argue that it may be the case that determinism fails under one formulation but reigns under the other. Whether this is so depends on our view on the nature of laws, and may also depend on the inter-theoretical relationships among our best physical theories, or so shall we argue.
\end{abstract}

Keywords Physical possibility $\cdot$ Determinism $\cdot$ Laws of nature $\cdot$ Best systems account of laws

\section{Introduction}

Determinism is a metaphysical doctrine stating that under certain conditions there is only one way how certain events or facts may be. Adopting the possible worlds approach for understanding modal statements, much of the philosophical literature focuses on the question whether our best physical theories are deterministic, that is whether the worlds that are physically possible according to these theories are deterministic:

(D) Let $\mathcal{W}$ be the set of physically possible worlds according to a physical theory $T$. A world $w \in \mathcal{W}$ is deterministic according to $T$ just in case for any $v \in \mathcal{W}$, if the state of $w$ and $v$ agrees at any time, then $w$ and $v$ agree at all times. The theory $T$ is deterministic just in case all $w \in \mathcal{W}$ are deterministic according to $T$.

Balázs Gyenis

gyepi@hps.elte.hu

1 Department of Philosophy, Logic, and Scientific Method, London School of Economics, London, UK

2 Institute of Philosophy, Research Centre for the Humanities, Hungarian Academy of Sciences, Budapest, Hungary 
Determinism of a theory is thus determined by its physically possible worlds: loosely speaking, the narrower their range, the better determinism's chances are.

To get a better grip on definition (D) - which is commonly accepted in the philosophy of science literature (see i.e. $[12,31]$ )—we should clarify the concepts of physically possible worlds, states, and physical laws of a physical theory. According to the received view of physical possibility the set of physically possible worlds of a theory is determined by the physical laws of the theory. The way how ([9], p. 412; [12], p. 13; [28], p. 18) cash out how this determination by physical laws happens is the following:

(a) A possible world is physically possible if and only if it satisfies the physical laws of the actual world,

or, resorting to (a)'s theory-relativized version:

(A) A possible world is physically possible according to a theory $T$ if and only if it satisfies the physical laws of $T$.

If we assume that $L$ is a physical law of $T$ if and only if $L$ is a physical law of the actual world, then the theory-relativized notion (A) is equivalent with its actual world-based (a) counterpart. Authors frequently introduce the notion of physical possibility through its actual world-based version, but in practice they invariably rely on the theory-relativized version, given the focus of the foundations of physics literature on implications of our current physical theories, our best attempts at getting an understanding of our actual world.

To fill in the remaining blanks we need to make further representational choices. For the sake of simplifying discussion let us

(1) settle with a precise definition of a solution of a differential equation that is a function of a time parameter, and agree that the so-defined solutions, and the so-defined solutions only, satisfy the differential equation,

(2) assume that the physical laws of $T$ are mathematically represented by a differential equation $E$,

(3) assume that a physical state is mathematically represented by an (initial) value of a solution of $E$ taken at some time, and

(4) assume that different solutions of $E$ represent different possible worlds.

Given (1)-(4), (A) and (D) the theory $T$ is deterministic if and only if

(5) all initial value problems of $E$ have at most one solution.

Determinism of a theory is known to be sensitive to assumptions (1)-(4) due to leeways in our choices of mathematical representation; an extensive discussion can be found in Earman [14] and in Gyenis [21] (see also [18] for a general, abstract approach to differential equations). Many physicists would be surprised 
to learn that, despite an effort to settle with representational choices for (1)-(4) that give best running chance for determinism, (5) typically fails for many if not for most of our best physical theories. As a consequence determinism fails according to many of our best physical theories, which is a common wisdom in the foundations of physics literature.

Can this conclusion fail to follow if we accept the received view of physical possibility, the commonly accepted determinism definition (D), and a representational choice akin to (1)-(4) that entails the failure of (5)? In this paper we point out that a different extant formulation of the received view of physical possibility may lead us to a different conclusion. There is a split in philosophers' understanding of how the set of physically possible worlds is determined by the physical laws. When they present the view they take to be standard, philosophers usually state one of two formulations, arguably without being conscious that they are different. The first is formulation (a) and resp. (A) we saw above. The second formulation, as professed by ([34], p. 6; [6], p. 174; [42], p. 62) and many other physicists and philosophers (see also [13,35] for a similar distinction), is the following:

(b) A possible world is physically possible if and only if it has the same physical laws as does the actual world.

(B) A possible world is physically possible according to a theory $T$ if and only if it has the same physical laws as does $T$.

Although in both (A) and (B) formulations it is the physical laws that determine the set of physically possible worlds, we point out that this set of worlds may not be the same for the two formulations: whether this is so depends on our conception of physical laws. If there is indeed a difference between the set of physically possible worlds of (A) and (B), then it may also be the case that while most of our physical theories are not deterministic under (1)-(4) and (A), they are deterministic under the same (1)-(4) and (B). We point out that some relatively standard conceptions of laws may entail this situation, and as a consequence we need to be more conscious about our choices regarding the notion of physical possibility and of laws.

To make it clear, this paper does not intend to defend or develop new philosophical concepts for determinism, physical possibility, laws of nature, alternative mathematical representations for states and laws, and neither does it aim to focus on determinism in classical mechanics despite illustrating the ideas through an example from that physical theory. On the contrary, all employed concepts are standard and explicitly adopted by the current philosophical literature (with the exception of the BATS account of laws about which we argue that it is also implicitly embraced by Humeans). This paper is raising consciousness that certain combination of otherwise standards concepts of physical possibility and laws of nature can allow us to challenge (given certain technical assumptions) the claim, commonly accepted as a conclusion in the philosophical literature, that our best physical theories are not deterministic. 


\section{Accounts of Laws of Nature}

There is a difference between the question 'what are physical laws' and the question 'what are the physical laws.' The latter may be answered by providing a list of laws that are supposedly represented by differential equations featured in our most revered physical theories; the former question asks about the criterion that differentiates laws that make their way to the list from other true propositions that are non-laws.

There is not only a plethora of accounts of laws of nature but also many ways to group and label them alongside commonly shared characteristics. Here we follow Cohen and Callender [10] in distinguishing three varieties.

The No-Laws camp believes that there are no laws of nature: in idealized models we may find causes, symmetries, or some general principles but they are not worthy to be elevated to the status of laws. These accounts are frequently driven by a sort of pessimistic meta-induction: ([40], p. 130, pp. 180, 181) draws motivation from the perceived failure of major approaches such as that of Lewis and Armstrong while ([20], pp. 86-91) cites cases from the history of science in which generalizations once thought to be laws were proven to be false. Mumford [30], Cartwright [8], and some projectivist accounts such as that of Ward [41] have also been lumped under the No-Laws heading. If one maintains that there are no laws, then one can not appeal to the laws to determine physical possibilities, and hence needs to reject the received view of physical possibility.

The Governing camp, advocates of which include Armstrong [1-4], Dretske [11], Maudlin [28], Shoemaker [37], Swoyer [38], and Tooley [39], insists that genuine laws of nature exist and stipulate that these laws govern events and happenings in the world. In the eloquent words of Cohen and Callender, "just as librarians enforce the rules of book borrowing and policemen enforce traffic rules, so some Governing theorists think that necessitarian relations, primitive accessibility relations, or primitive universals enforce certain behaviors upon the events of the world. Other advocates of Governing are silent on how the laws manage these feats, but insist that they do and treat laws with the requisite governing powers as primitive" (ibid. p. 2). Not only do some Governing advocates treat laws as primitive, but they also ascribe the power to the laws to generate, evolve, bring about, or propagate physical states. Thus ([28], p. 15) writes:

My own proposal is simple: laws of nature ought to be accepted as ontologically primitive. We may use metaphors to fire the imagination: among the regularities of temporal evolution, some, such as perhaps that described by Schrödinger's equation, govern or determine or generate the evolution.

The Non-Governing camp also insists that genuine laws of nature exists but deny that these laws govern. Instead of governing the laws merely describe certain aspects of patterns that obtain in the mosaic of events. One of the most prevalent Non-Governing views is the Best System account associated with Callender [5], Cohen and Callender [10], Earman [12], Lewis [22-25], Mill [29], Loewer [26] and Ramsey [36]. According to the Best System account the distinguishing 
feature of laws is that they are highly informative about the world in a simple way: they are propositions of a deductive system which best balances informativeness and simplicity.

One of the main dividing lines between the Governing and the Non-Governing camps is in how they relate to Humean supervenience, "the doctrine that all there is in the world is a vast mosaic of local matters of particular fact, [...] an arrangement of qualities. And that is all. There is no difference without difference in the arrangement of qualities. All else supervenes on that." (Lewis; [24], pp. ix, x). Humean supervenience tries to capture the empiricist constraint that laws should supervene on the mosaic of non-nomic facts; what counts as 'non-nomic' and 'fact' is a matter of debate. There exist several, more sophisticated statements of Humean supervenience; for an analysis see Earman and Roberts [16, 17]. In general advocates of a Governing view deny Humean supervenience about laws of nature, while advocates of Non-Governing views, in particular of the Best System view, embrace it.

All three accounts of laws have been severely criticized. Without laws it is very difficult to explain practice of the physical sciences and to explain why certain regularities and patterns among events hold. While Governing views may be able to explain, in terms of their favored necessitarian relations, primitive accessibility relations, or primitive universals, why certain patterns among the events hold they seem to merely push the explanatory issue one step back as they are unable to explain why the employed particular relations or universals hold. If they provide no explanatory advantage, then postulating laws as entities existing in addition to the mosaic of facts goes against metaphysical parsimony. The Best System view is in danger to render laws subject dependent as notions of simplicity, informativeness, and best balance seem to be very difficult to articulate from an objective point of view. For an overview of the main advantages and the main lines of criticism mounted against these views see i.e., Carroll [7].

In their above-mentioned article Callender and Cohen proposed to relativize the Best System account to specific choices of basic kinds or basic predicates. They claim that even though there is indeed no objective point of view from which simplicity, informativeness and best balance could be judged, such vantage point is also not necessary to find a satisfactory account of laws. They hold a true generalization to be a law relative to a choice of basic kinds or predicates if the generalization appears in all immanently Best Systems relative to these basic kinds or predicates. They take relativity to be an advantage of their account; with its help they claim to be able to solve many problems plaguing the traditional Best System view, such as the problem of immanent comparisons. In addition they see Relativized Best System or RBS to be capable of giving an account for laws in the special sciences.

This last point will be of interest for us later. In order to accommodate the special sciences, the authors concede that RBS needs to allow for exceptions because claims of special sciences "will be in principle defeatable by lower-level physical limitations" (ibid. p. 25). One proposal they make to amend this difficulty is to "relax the requirement that the MRL [Mill-Ramsey-Lewis, referred here as Best System] laws be true and replace it with some other requirement, like pragmatic reliability (as determined by the science of interest)" (ibid. fn 24). Apparently the authors believe that (exact) truth is not a sine qua non of laws: it is consistent with an understanding 
of laws as simple and informative systematizations that under certain circumstances laws may happen to be false. Presumably this can only happen if the price of falsehood is paid back in improvement of the overall balance of simplicity and informativeness. As generalizations that are not even approximately true can not be informative, this suggestion amounts to relaxing the condition that laws are true generalizations to the condition that laws are approximately true generalizations. For later purposes we can thus label this the Best Approximately True Systems (BATS) account:

(BTS) Laws are true propositions of the deductive systems which best balance simplicity and informativeness.

(BATS) Laws are approximately true propositions of the deductive systems which best balance simplicity and informativeness and approximation to truth.

Difference between BTS and BATS may be highlighted by an example. Which deductive system carries more the merit of lawhood: one which implies the exact value of the electromagnetic field in one spacetime point but is completely silent about its values everywhere else, or another which implies the values of the electromagnetic field everywhere, but only up to an approximation which lies beyond our measurement capabilities to detect? BTS would force us to choose the first option while BATS would also allow for the second.

We do not aim to argue here for the validity of BATS. We merely submit that BATS is an account which should be amenable to defenders of a Best System account of laws. As we have already pointed out the Relativized Best System account implicitly already embraces BATS. Any other account that allows the special sciences to have laws is also likely to implicitly embrace BATS: if the same mosaic of events may be subject to laws of theories located on different levels of the proverbial layer cake, then truth with approximation should be sufficient for laws, since theories on a higher level typically operate with more coarse-grained descriptions than theories on the lower level that have more nuanced descriptive tools.

\section{Received Views and Possible Worlds}

The set of physically possible worlds under formulation (B) may be narrower than under formulation (A). Formulation (B) not only requires a physically possible world to satisfy the physical laws $L$, but also that $L$ are the physical laws of the physically possible worlds themselves. This latter condition does not follow automatically from the former: depending on our conception of physical laws it may be the case that a possible world $w$ satisfies $L$, but $L$ is not a physical law in $w$.

Indeed for a Best System account we should expect such cases to occur. Let our theory $T$ be classical electrodynamics. Since the possible world which is empty also satisfies Maxwell's equations, it is physically possible according to formulation (A). But this empty world may not be physically possible according to formulation (B) and a Best System account, since the deductive system best balancing 
informativeness and simplicity in the empty world may simply contain the proposition that 'the world is empty.' Maxwell's equations are true in this empty world, but they are not laws of it, since they are not simple enough given the alternatives.

The difference can also be easily seen without invoking a Best System account, as long as we maintain Humean supervenience about laws. Most of our current physical theories allow for an "empty" world to satisfy its physical laws. Hence the empty world is physically possible according to several different theories under formulation (A). However, this empty world could possess exactly the same laws of at most one of these theories, and hence it can not be physically possible under formulation (B) for the other theories. Hence as long as our account of laws of nature is capable to recover at least some of our current physical theories (without which it'd arguably be without much merit as an account of laws), the two formulations will produce different physically possible worlds.

\section{Must Determinism Fail? A Research Project}

Suppose now that assumptions (1)-(4) hold for a theory $T$ with differential equation $E$ but (5) does not. Then it follows from formulation (A) of the received view and from definition (D) that $T$ is not deterministic. Is there an account of laws of nature which, conjoined with formulation (B) of the received view, renders $T$ deterministic according to (D)?

The set of physically possible worlds of formulation (A) is the union of the set of solutions of $E$ that are deterministic and the set of solutions of $E$ that are not deterministic. In order to achieve determinism the set of physically possible worlds of formulation (B) must be narrower: it may contain all deterministic solutions, but it can not contain more than one solution from any of the 'non-determinism bouquets' (from any of the sets of solutions of $E$ that agree in some of their states). We are thus seeking an account of law of nature which crowns $E$ as the representation of the law of possible worlds that are represented by deterministic solutions, but which crowns $E$ as the representation of the law of at most one of the possible worlds that are represented by solutions in a non-determinism bouquet.

What properties may tell apart deterministic and not deterministic solutions? The following difference is clear: for deterministic solutions $E$ is maximally informative in the sense that being supplemented by a state it furnishes all non-nomic facts. $E$ however is not maximally informative in this sense for a not deterministic solution, and in fact may fare pretty badly in narrowing the set of non-nomic facts that may obtain. This difference in informativeness of $E$ for deterministic and not deterministic solutions signals that some variant of the Best Systems account of laws may do the job for us.

An account of laws that exploits the difference in informativeness of $E$ between deterministic and not deterministic solutions may help ensuring determinism of the theory. Suppose that $s$ is a not deterministic solution of $E$ : albeit $s$ satisfies $E, E$ is not necessarily a law of the world represented by $s$, because there may be an alternative proposition (not necessarily a differential equation) $L^{\prime}$ that our account of laws crowns as the law of the world represented by $s$ on the grounds that $L^{\prime}$ is more 
informative of $s$ than $E$. If such an alternative $L^{\prime}$ exists, then $s$ is not physically possible according to the (B) formulation of physical possibility, since the (B) formulation requires a physically possible world to have the same laws as the theory, but $E$ is not a law of $s$. To generalize, if for any not deterministic solution there existed an alternative proposition that is crowned as the law of said not deterministic solution, then determinism of the theory would be restored. If the account of laws is Best Systems type then the alternative proposition $L^{\prime}$ would need to provide a better balance of informativeness and simplicity than does the law represented by $E$. To sum up, if for any not deterministic solution such an alternative $L^{\prime}$ existed, then the Best Systems view, together with assumptions (D), (1)-(4), $\neg(5)$ and formulation (B) would entail that the theory $T$ is deterministic, despite $T$ not being deterministic under assumptions (D), (1)-(4), $\neg(5)$ and formulation (A).

This problem defines a research project whose success would entail the nonfailure of determinism. On the mathematical-physical side of the project we would inquire whether for every not deterministic solution of a physically relevant differential equation we could find another simple proposition that is more informative about this solution than the original differential equation. On the philosophical side of the project we would seek to independently motivate the notions of simplicity and informativeness that characterize said proposition, and on the basis of these notions we would seek the variant of the Best Systems view which crowns this and such propositions as laws of the corresponding not deterministic solutions. We now take a look at the plausibility of success of this research project to save determinism.

\subsection{Could Our Research Project Plausibly Succeed?}

In abstracto the prospects of our research project of saving determinism seem bleak. For a simple example consider John Norton's Dome as analyzed by Norton [32, 33] and Malament [27]. Imagine a ball resting on the top of a carefully designed Domeshaped surface. The ball can move frictionlessly, but it is restricted to move on the surface, and is only influenced by a homogeneous gravitational field. Our physical theory $T$ is classical mechanics with Newton's laws, in particular the second law: $F=m a$. In order to find how the ball moves we need to solve the initial value problem, where the force $F$ of Newton's law is determined by the shape of the Dome and by the gravitational field, and where the initial values are the initial position and momentum of the ball. If this were a commonplace problem in classical mechanics we would get a unique solution telling us how the ball moves. However the shape of the Dome is trickily designed so that our initial value problem yields many different solutions: the ball spontaneously starts to roll from the top of the Dome, but classical mechanics can't tell us when this starting moment happens. In other words, the Dome shows that under the assumptions (1)-(4) property (5) fails in classical mechanics. Given definition (D) and formulation (A) the solutions of the Dome are not deterministic, and hence we get the somewhat counterintuitive conclusion that determinism fails in classical mechanics. Thus for the research project to succeed we ought to find, for each not deterministic solution of the Dome, a proposition that 
provides a better balance of simplicity and informativeness about the solution than $F=m a$.

Substituting the force determined by the shape of the Dome and by the gravitational field, Newton's law in the Dome world becomes

$$
x^{(2)}=\sqrt{x},
$$

where $x$ is the distance of the ball from the top of the Dome on its surface and the ${ }^{(2)}$ superscript denotes the second derivative with respect to the time parameter. (As the reader can readily check, the family of "Dome solutions",

$$
x(t)= \begin{cases}0 & \text { if } 0 \leq t \leq \tau \\ \frac{1}{144} t^{4} & \text { if } \tau<t,\end{cases}
$$

parametrized with the time $\tau$ when the ball spontaneously starts to roll from the top of the Dome, all solve equation 1 for the same initial values $x(0)=0, x^{(1)}=0$. A particular choice of $\tau$ yields a particular Dome solution.)

Albeit equation (1) contains some "ugly" mathematical notation it may strike us as "simple". Can we really hope to find a proposition which better balances simplicity and informativeness for a particular not deterministic Dome solution than the equation (1) itself?

Well, simplicity is in the eye of the beholder. Contrast (1) with the following differential equation:

$$
x^{(k)}=0
$$

where $k$ is a yet undetermined number. A charitable reader is going to agree that, at least at a first blush, it is not unreasonable to hold that equation (2) is "simpler" than equation (1). There may be issues with the number $k$ not being "simple" enough, but (2) is homogenous and only makes reference to differentiation, while (1) is inhomogeneous and involves, besides differentiation, the square root of the variable as well.

The solutions of equation (2) are polynomials up to the $k$-th degree. Polynomials can be used to approximate finite trajectories and thus they can approximately describe the path the ball rolling down on the Dome takes in any particular Dome solution. Mathematically speaking for any finite stretch of a solution $s$ of equation (1) we can find a value for $k$ so that a solution $s^{\prime}$ of equation (2) stays within our desired level of approximation to $s$. By appropriately choosing $k$ number of initial values we can uniquely determine this $s^{\prime}$ solution. Thus, assuming our Best System only systematizes a finite lifespan of the Dome universe, equation (2) may count as a proposition of a deductive system which approximates truth, is informative and is simple.

As equation (2) is merely approximately true while equation (1) is exactly true of a Dome solution, an account of laws that crowns (2) as a law for such a solution would need to allow approximate truth to be sufficient for lawhood. The Best Approximately True Systems is such an account. If we accept that equation (2) is simpler than equation (1), it becomes a question of balance whether (2) or (1) should count as a law in a Dome solution according to BATS. As the loss in truth due to the 
approximation could be made arbitrarily small, if a gain in simplicity and informativeness has an effect on the balance of informativeness, simplicity and approximation to truth, then the overall balance may be improved by a suitable choice of small approximation. In case it turns out that equation (2) provides a better balance than equation (1), we get the conclusion that in formulation (B) of physical possibility a not deterministic Dome solution is not physically possible according to classical mechanics, since its laws are not those of Newton's. The laws of deterministic solutions of $F=m a$ would however be still those of Newton's, since for deterministic solutions we would loose informativeness by moving to an approximation like (2). Thus deterministic solutions are physically possible according to classical mechanics, while not deterministic solutions are not.

This conclusion does not depend on the assumption of having only one instance of the Dome-particle system being present in the world. Taking $x$ to be a $3 N$-dimensional vector equation (2) applies to a system of $N$ point particles and hence it has solutions approximating any finite lifespans of worlds that contain, say, $N$ number of Dome-particle systems, without losing any simplicity or informativeness. Nevertheless the argument has many shortcomings, notably the assumption that our Best System only systematizes a finite lifespan of the possible world. The gain in simplicity by equation (2) might turn out to be too small to favor one system over the other, and it may even be illusory. The distance measured between trajectories by the supremum norm might not be physically relevant. A further nagging point is that equation (2) requires $k$ number values to determine the solution instead of equation (1)'s 3 (two plus the time when the ball starts to spontaneously roll), their only advantage being that all $k$ of them are initial values. This brings up the question whether informativeness or simplicity of laws should depend upon their ability to get combined with additional accessible informative and simple propositions in order to produce further informative and simple propositions about the world (for an elaboration on this point, see [21]).

In general, instead of attempting to find some abstract scheme to approximate not deterministic solutions of arbitrary differential equations, it seems worthwhile to investigate the relationship of various concrete differential equations in physics. Among the partial differential equations the typical sources of failure of uniqueness are the so-called parabolic and elliptic equations, such as the classical heat equation or the Laplace equation. There is a general sense in which such equations can be approximated by quasilinear first order hyperbolic equations whose initial value problems have a unique solution. Robert Geroch, one of the main authorities on partial differential equations in physics opines that

A case could be made that, at least on a fundamental level, all the "partial differential equations of physics" are hyperbolic - that, e.g. elliptic and parabolic systems arise in all cases as mere approximations of hyperbolic systems. Thus, Poisson's equation for the electric potential is just a facet of a hyperbolic system, Maxwell's equations. ([19], pp. 2, 3)

Geroch then proceeds to show that a general symmetrization procedure is available for quasilinear first order hyperbolic systems; for symmetric systems general theorems on existence and uniqueness of solutions are available. 
If conversely a not deterministic solution $s$ of the parabolic or elliptic equation $E$ of a physical theory $T$ can be approximated by a deterministic solution $s^{\prime}$ of a hyperbolic equation $E^{\prime}$ of another physical theory $T^{\prime}$ then physics, beyond helping with the problem of rendering the theory $T$ deterministic, may also help to alleviate the philosophical problem of vagueness plaguing the key concepts-informativeness, simplicity, and approximation—of the Best Approximately True Systems account of laws. As $E^{\prime}$ represents a law of another physical theory, it is likely going to pass both as simple and informative. Having unique initial value problems, $E^{\prime}$ is also likely going to be more informative than $E$. Moreover, since the main motivation for upholding simplicity and informativeness as defining characteristics of laws is that these seem to be true of the fundamental equations that appear in our physical theories, we may even bypass the problem of having to define what we mean by informativeness and simplicity altogether. The sense in which $s$ and $s^{\prime}$ approximate each other is made explicit by the mathematical claim of the approximative relationship itself, and this sense of approximation can also provide the relevant notion of approximation to truth required by the Best Approximately True Systems account of laws.

This analysis is cursory, but it adds yet an additional reason for why thorough investigation of the approximative relationships of different physical theories should further be pursued. The prospects of our research project to save determinism could only be adequately judged after such detailed investigation is brought to fruition.

\section{Concluding Remarks}

This paper raises awareness that determinism of theories crucially depends on our understanding of physical possibility and of laws of nature, and argues that a combination of extant philosophical accounts of physical possibility and laws of nature could imply that certain physical theories (which are widely regarded as not deterministic in the philosophical literature) are deterministic after all. Although our focus on determinism of theories is in line with the general discussion of determinism in the foundations of physics literature, one could argue that our primer philosophical interest lies in the truth of determinism of our actual world, and it is not a foregone conclusion that (failure of) determinism of current physical theories has any implication about (failure of) determinism of our actual world. First, although the theory-based formulations of physical possibility ((A) and (B)) are equivalent with their actual world-based counterparts ((a) and (b)) in case we consider the theory $T_{a}$ which comprises all and only those laws that are laws in the actual world, there are good reasons to believe that none of our current physical theories express the same laws as $T_{a}$; worse, the actual world might not be, strictly speaking, physically possible according to any of our current physical theories. This limits the value of the insights we can gain about determinism of our actual world from studying the fate of determinism of our current physical theories. Second, we should especially be careful in drawing conclusions from failure of determinism of a theory to failure of determinism of the actual world. A theory fails to be deterministic if any of its physically possible worlds fails to be deterministic; failure of determinism of some 
physically possible worlds however does not entail that the actual world also fails to be deterministic. In principle a theory could fail to be deterministic and yet the same theory could be suggestive of the truth of determinism of the actual world. Hence even if we rejected the combination of the account of physical possibility and laws of nature that we argued could avert the failure of determinism of theories, it could still be the case that our actual world $a$ is deterministic even if its complete and true $T_{a}$ physical theory is not deterministic.

This being said failure of determinism of a theory may still be indicative of serious problems, especially when there is another theory describing the same phenomena that is deterministic. Although there have been recent attempts to explore how failure of determinism of one theory may be cured by another (see [15]), the literature still assumes that determinism of a particular physical theory can be assessed by addressing that theory alone. If an investigation of inter-theoretical relationships would succeed along the lines proposed in the previous section then, with formulation (B) of the received view of physical possibility and with a Best Approximately True Systems account of laws, determinism of a particular physical theory also becomes dependent upon its relationship to other physical theories. Thus a physical theory that is not deterministic with formulation (A) may turn out to be deterministic according to formulation (B).

The subtle difference between the (A) and (B) formulation of the received view of physical possibility could lead to substantially different philosophical conclusions regarding the fate of determinism, and hence the question naturally arises which of these formulations should be adopted. We have not investigated this question here, and it is unclear whether we can enlist any independent reasons that could strongly compel us to favor one formulation of the received view over the other (for details see [21]). However, if determinism is indeed to be rather thought of as a heuristic, 'defeasible methodological imperative' ([14], p. 1372), then the formulation of the received view of physical possibility and the account of laws of nature whose combination succeeds in salvaging determinism does seem to have an advocate at the trial of grand metaphysical speculations.

Acknowledgements All thanks expressed in the preface of Gyenis [21], the (unpublished) dissertation whose Chapter 2 formed the basis of this paper, carries over to this article as well. I would also like to thank the comments and suggestions of two anonymous reviewers, one of whom wrote unusually thorough and helpful reviews. (Of course, all remaining errors are mine.) This research has been supported by the National Research, Development and Innovation Office K-115593 Grant.

Open Access This article is licensed under a Creative Commons Attribution 4.0 International License, which permits use, sharing, adaptation, distribution and reproduction in any medium or format, as long as you give appropriate credit to the original author(s) and the source, provide a link to the Creative Commons licence, and indicate if changes were made. The images or other third party material in this article are included in the article's Creative Commons licence, unless indicated otherwise in a credit line to the material. If material is not included in the article's Creative Commons licence and your intended use is not permitted by statutory regulation or exceeds the permitted use, you will need to obtain permission directly from the copyright holder. To view a copy of this licence, visit http://creativecommons.org/licen ses/by/4.0/. 


\section{References}

1. Armstrong, D.: A Theory of Universals. Cambridge University Press, Cambridge (1978)

2. Armstrong, D.: What Is a Law of Nature?. Cambridge Univerity Press, Cambridge (1983)

3. Armstrong, D.: What makes induction rational? Dialogue 30, 503-511 (1991)

4. Armstrong, D.: The identification problem and the inference problem. Philos. Phenomenol. Res. 53, 421-422 (1993)

5. Callender, C.: What makes time special. http://fqxi.org/data/essay-contest-files/Callender_FQX.pdf (2008). Accessed 14 Feb 2009

6. Carroll, J.W.: Laws of Nature. Cambridge University Press, Cambridge (1994)

7. Carroll, J.W.: Laws of nature. In: Zalta, E.N. (ed.) The Stanford Encyclopedia of Philosophy, spring 2012 edn (2012)

8. Cartwright, N.: How the Laws of Physics Lie. Oxford University Press, New York (1983)

9. Chisholm, R.M.: He could have done otherwise. J. Philos. 64, 409-417 (1967)

10. Cohen, J., Callender, C.: A better best system account of lawhood. Philos. Stud. 145, 1-34 (2009)

11. Dretske, F.: Laws of nature. Philos. Sci. 44, 248-268 (1977)

12. Earman, J.: A Primer on Determinism. Reidel, Dordrecht (1986)

13. Earman, J.: Bangs, Crunches, Whimpers, and Shrieks: Singularities and Acausalities in Relativistic Spacetimes. Oxford University Press, Oxford (1995)

14. Earman, J.: Aspects of determinism in modern physics. In: Butterfield, J., Earman, J. (eds.) Handbook of the Philosophy of Science. Philosophy of Physics, Part B. Elsevier, Amsterdam (2007)

15. Earman, J.: Essential self-adjointness: implications for determinism and the classical-quantum correspondence. Synthese 169, 27-50 (2009)

16. Earman, J., Roberts, J.: Contact with the nomic: a challenge for deniers of Humean supervenience about laws of nature (part I). Philos. Phenomenol. Res. 71, 1-22 (2005a)

17. Earman, J., Roberts, J.: Contact with the nomic: a challenge for deniers of Humean supervenience about laws of nature (part II). Philos. Phenomenol. Res. 71, 253-286 (2005b)

18. Fattorini, H.O.: The Cauchy Problem. Cambridge University Press, Cambridge (1983)

19. Geroch, R.: Partial differential equations of physics. arXiv:9602055v1 (2008)

20. Giere, R.: Science Without Laws. University of Chicago Press, Chicago (1999)

21. Gyenis, B.: Well posedness and physical possibility. Ph.D. Dissertation, Department of History and Philosophy of Science, University of Pittsburgh (2013)

22. Lewis, D.: Counterfactuals. Harvard University Press, Cambridge (1973)

23. Lewis, D.: New work for a theory of universals. Australas. J. Philos. 61, 343-377 (1983)

24. Lewis, D.: Philosophical Papers, vol. II. Oxford University Press, New York (1986)

25. Lewis, D.: Humean supervenience debugged. Mind 103, 473-490 (1994)

26. Loewer, B.: Humean supervenience. Philos. Top. 24, 101-126 (1996)

27. Malament, D.: Norton's slippery slope. Philos. Sci. 75(4), 799-816 (2008)

28. Maudlin, T.: The Metaphysics Within Physics. Oxford University Press, New York (2007)

29. Mill, J.: A System of Logic. Longmans, Green and Co., London (1947)

30. Mumford, S.: Laws in Nature. Routledge, London (2004)

31. Müller, T., Placek, T.: Defining determinism. Br. J. Philos. Sci. 69, 215-252 (2018)

32. Norton, J.: Causation as folk science. Philos Imprint 3(4), 1-22 (2003)

33. Norton, J.: The domeaunexpectedly simple failure of determinism. In: Proceedings of the 2006 Biennial Meeting of the Philosophy of Science Association, Philosophy of Science (2006)

34. Bradley, R., Swartz, N.: Possible Worlds: An Introduction to Logic and Its Philosophy. Hackett Publishing Company, Indianapolis (1979)

35. Roberts, J.: Lewis, Carroll, and seeing through the looking glass. Australas. J. Philos. 76, 426-438 (1998)

36. Ramsey, F.: Foundations. Routledge and Kegan Paul, London (1978)

37. Shoemaker, S.: Causal and metaphysical necessity. Pac. Philos. Q. 79, 59-77 (1998)

38. Swoyer, C.: The nature of natural laws. Australas. J. Philos. 60, 202-223 (1982)

39. Tooley, M.: The nature of laws. Can. J. Philos. 7, 667-698 (1977)

40. van Fraassen, B.: Laws and Symmetry. Clarendon Press, Oxford (1989)

41. Ward, B.: Humeanism without humean supervenience: a projectivist account of laws and possibilities. Philos. Stud. 107, 191-218 (2002) 
42. Witmer, D.G.: Sufficiency claims and physicalism. In: Gillett, C., Loewer, B. (eds.) Physicalism and Its Discontents. Cambridge University Press, Cambridge (2001)

Publisher's Note Springer Nature remains neutral with regard to jurisdictional claims in published maps and institutional affiliations. 